\title{
Utilidad de la cardiorresonancia magnética en una unidad de cardiopatías familiares de reciente implantación. Resultados a corto plazo
}

\author{
Cardiovascular magnetic resonance (CMR) implementation in a newly-established \\ cardiomyopathies unit
}

\author{
Juan A. Requena-Ibáñez ${ }^{*}$, Jesús Piqueras-Flores ${ }^{1}$, Miguel Rienda-Moreno $^{2}$, Ramón Maseda-Uriza ${ }^{1}$, \\ Pedro Pérez-Díaz y Raquel Frías-García ${ }^{1}$ \\ ${ }^{1}$ Servicio de Cardiología; ${ }^{2}$ Servicio de Radiología. Hospital General de Ciudad Real, Ciudad Real, España
}

La resonancia magnética cardíaca (cardiorresonancia) se ha convertido en una técnica imprescindible en el estudio de las miocardiopatías. Posee múltiples ventajas que explican su creciente papel en la práctica clínica. Ofrece imágenes de alta resolución espacial y temporal, y valora de forma integral la función y la anatomía cardíacas. No requiere el uso de radiación ionizante. Es una técnica de imagen con capacidad multiplanar, libre de asunciones geométricas y permite estudiar el corzón y los grandes vasos sin las limitaciones propias de la ventana acústica ${ }^{1}$. En la actualidad es la técnica más precisa y reproducible para el estudio de la función sistólica biventricular global y regional².

Además, puede caracterizar el tejido miocárdico para efectuar una aproximación etiológica y valorar la presencia de fibrosis miocárdica. Todo ello confiere a esta prueba un valor indudable en el diagnóstico y la gradación del pronóstico de este tipo de pacientes.

Nuestro objetivo es evaluar la utilidad de la cardiorresonancia en una unidad de cardiopatías familiares de reciente implantación. Desde enero de 2017 hasta octubre de 2018283 pacientes fueron evaluados. En los casos que no tenían contraindicaciones, en los que los resultados de otras pruebas diagnósticas eran discordantes o era necesario un estudio de caracterización tisular o función ventricular más exacto, se solicitó una cardiorresonancia. La disponibilidad del equipo de resonancia también fue determinante en este centro. Al final se realizó en 83 pacientes.

La sospecha diagnóstica fue miocardiopatía hipertrófica (32 pacientes), seguida de ventrículo izquierdo no compactado (17) y miocardiopatía dilatada (14). Menos frecuente fueron la miocardiopatía arritmogénica (6), síndrome de Marfan y afectación aórtica (5), amiloidosis cardíaca (3) y miocardiopatía restrictiva (1).

Para calcular volúmenes, masa, fracción de expulsión y grosor parietal se utilizaron secuencias b-SSFP (cine balanced steady state free precesion), por lo que son la principal fuente de información anatómica y funcional. Las características que hacen ideales a las secuencias b-SSFP para estudiar el corazón son su gran velocidad de adquisición, alta relación señal-ruido y el gran contraste nativo (sin necesidad de medio de contraste) entre la sangre, el miocardio y la grasa.

Para estudiar el fenómeno de realce tardío se necesitan medios de contraste de gadolinio de

\section{Correspondencia:}

*Juan A. Requena-Ibáñez

E-mail: juan.antonio.requena.ib@gmail.com DOI: 10.24875/ACM.20000136 CC BY-NC-ND (http://creativecommons.org/licenses/by-nc-nd/4.0/).

Disponible en internet: 14-09-2020 Arch Cardiol Mex. 2020;90(3):363-365 www.archivoscardiologia.com 
distribución extracelular, que aumentan la sensibilidad de la RM para detectar lesiones y mejoran la caracterización tisular.

El gadolinio es un metal con una elevada capacidad paramagnética y máxima capacidad de atracción de los neutrones, lo que lo convierte en un buen medio de contraste. Existen diferentes quelatos de gadolinio en el mercado, con ligeras diferencias farmacológicas entre ellos. En este centro en particular se utilizó gadoterato de meglumina a una dosis de $3 \mathrm{ml} / \mathrm{kg}$, de estructura macrocíclica y carga iónica, características estas últimas que mejoran la estabilidad de la molécula.

El gadolinio tiene una distribución extracelular y se difunde libremente a través de los capilares. Se acumula en el espacio intersticial de las áreas en las que existe daño en la integridad de la membrana celular o aumento del espacio extracelular, ya sea por necrosis, inflamación o fibrosis. De esta forma, el realce tardío representa una acumulación anormal de contraste en un tejido (realce) que persiste en un período prolongado de tiempo (tardío), alrededor de 25 minutos después de la inyección intravenosa del bolo de contraste.

Para ello se emplearon secuencias potenciadas en $\mathrm{T} 1$ derivadas de las anteriores (b-SSFP), a las que se añadió un pulso de inversión capaz de anular la señal del miocardio sano (hipointenso) y así exacerbar el contraste con las zonas de realce patológico (hiperintenso).

Del total de casos solicitados, 60 correspondieron a varones, 57 a casos índice y 26 a casos de estudio familiar. La sospecha inicial se confirmó con cardiorresonancia en 63. Los más frecuentes fueron los siguientes: 27 pacientes con miocardiopatía hipertrófica, 11 con miocardiopatía dilatada, 10 con miocardiopatía no compactada y 5 con miocardiopatía arritmogénica. Por otra parte, el estudio de cardiorresonancia fue normal en 11 sujetos, lo cual posibilitó el alta definitiva de 8 pacientes, sobre todo por hipertrabeculación ventricular sin criterios de ventrículo izquierdo no compactado, sospecha de miocardiopatía arritmogénica sin evidencia de alteraciones estructurales o funcionales y aumento del grosor miocárdico sin criterios de hipertrofia ventricular.

En 25 de los estudios se detectó realce tardío de gadolinio y en dos de ellos el resultado de la cardiorresonancia fue determinante para el implante del DAI. Se trataba de un caso de miocardiopatía dilatada con ventrículo izquierdo no compactado y disfunción ventricular grave y un caso de miocardiopatía hipertrófica familiar. La presencia de realce tardío representa un peor pronóstico y mayor riesgo en múltiples cardiopatías $^{3,4}$. Si bien la decisión de implantar un DAI depende en la actualidad del valor de la $\mathrm{FEVI}^{5}$, la cardiorresonancia ayudó a mejorar la estratificación de riesgo en estos pacientes y por tanto a racionalizar mejor su implante.

En general, los pacientes con realce tardío de gadolinio muestran en grado significativo menor fracción de expulsión (47 vs. 55\%, $p=0.014$ ). Para ello se utilizó la prueba $\mathrm{T}$ para muestras independientes.

Por último, se solicitó estudio genético a 63 pacientes. Todos ellos presentaban un resultado patológico en la cardiorresonancia (en particular aumento del grosor parietal en grado de hipertrofia). Las mutaciones identificadas más a menudo afectaban a los genes sarcoméricos, 11 MYBPC3 (Arg502Gln, Arg495Gln y Glu542GIn) y 5 con TTN Cys3529Ser. Como es habitual en los estudios genéticos, también se reconocieron mutaciones no claramente causales (dos mutaciones de significado incierto y tres mutaciones a estudio de cosegregación).

En conclusión, la experiencia en este centro concuerda con la tendencia actual de la cardiorresonancia en su consolidación como técnica de imagen complementaria a la ecocardiografía en el estudio de las miocardiopatías. La cardiorresonancia permite, junto con un adecuado enfoque clínico, acotar el diagnóstico diferencial, mejorar la estratificación del riesgo Eliminar esta palabra y guiar las decisiones clínicas ${ }^{6}$.

\section{Financiamiento}

$$
\text { Ninguno. }
$$

\section{Conflicto de intereses}

Sin conflictos de interés que declarar.

\section{Responsabilidades éticas}

Protección de personas y animales. Los autores declaran que para esta investigación no se han realizado experimentos en seres humanos ni en animales.

Confidencialidad de los datos. Los autores declaran que en este artículo no aparecen datos de pacientes.

Derecho a la privacidad y consentimiento informado. Los autores declaran que en este artículo no aparecen datos de pacientes. 


\section{Bibliografía}

1. Grothues F, Smith GC, Moon JC, Bellenger NG, Collins P. Comparison of interstudy reproducibility of cardiovascular magnetic resonance with two-dimensional echocardiography in normal subjects and in patients with heart failure or left ventricular hypertrophy. Am J Cardiol. 2002;90:29-34

2. San Román JA, Candell-Riera J, Arnold R. Análisis cuantitativo de la función ventricular izquierda comoherramienta para la investigación clínica. Fundamentos y metodología. Rev Esp Cardiol. 2009;62(5):535-51. Elliott P, Andersson B, Arbustini E. Classification of the cardiomyopathies: a position statement from the European Society Of Cardiology Working Group on Myocardial and Pericardial Diseases. Eur Heart J. 2008;29(2):270-276.
3. Heidary S, Patel H, Chung J. Quantitative tissue characterization of infarct core and border zone in patients with ischemic cardiomyopathy by magnetic resonance is associated with future cardiovascular events. $J$ Am Coll Cardiol. 2010;55(24):2762-2768.

4. Green JJ, Berger JS, Kramer CM, Salerno M. Prognostic value of late gadolinium enhancement in clinical outcomes for hypertrophic cardiomyopathy. JACC Cardiovasc Imaging. 2012;5(4):370-377.

5. Elliott P. Cardiomyopathy. Diagnosis and management of dilated cardiomyopathy. Heart. 2000;84(1):106-112.

6. De Smet K, Verdries D, Tanaka K. MRI in the assessment of non ischemic myocardial diseases. European J Radiol. 2012;81(7): 1546-1548. 